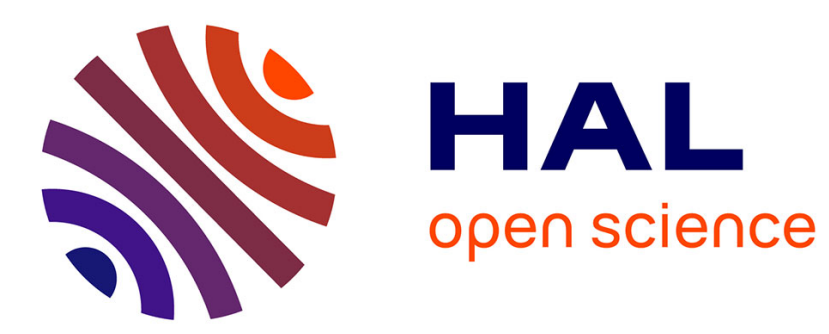

\title{
Ultrasound monitoring of bubble size and velocity in a fluid model using phased array transducer
}

\author{
Naïm Samet, Pierre Maréchal, Hugues Duflo
}

\section{To cite this version:}

Naïm Samet, Pierre Maréchal, Hugues Duflo. Ultrasound monitoring of bubble size and velocity in a fluid model using phased array transducer. NDT \& E International, 2011, 44 (7), pp.621-627. 10.1016/j.ndteint.2011.06.005 . hal-03313307

\section{HAL Id: hal-03313307}

\section{https://hal-normandie-univ.archives-ouvertes.fr/hal-03313307}

Submitted on 3 Aug 2021

HAL is a multi-disciplinary open access archive for the deposit and dissemination of scientific research documents, whether they are published or not. The documents may come from teaching and research institutions in France or abroad, or from public or private research centers.
L'archive ouverte pluridisciplinaire HAL, est destinée au dépôt et à la diffusion de documents scientifiques de niveau recherche, publiés ou non, émanant des établissements d'enseignement et de recherche français ou étrangers, des laboratoires publics ou privés. 


\title{
Ultrasound monitoring of bubble size and velocity in a fluid model
}

\section{using phased array transducer}

\author{
Naïm Samet, Pierre Maréchal, Hugues Duflo \\ Laboratoire Ondes et Milieux Complexes, LOMC FRE CNRS 3102, FANO FR CNRS 3110 , \\ Université du Havre, Place Robert Schuman, 76610 Le Havre, France. Tel/Fax: +332 327447 38/19 \\ naim.samet@univ-lehavre.fr
}

\begin{abstract}
Experimental studies on composite materials highlighted the existence of gas bubbles (voids) at different scales (micro and macro). These voids, resulting from the fabrication process, are sources of weakness for the end-user material. Therefore, several studies focus on the evaluation and the minimization of void rate. During injection of resin into the fibrous matrix, bubbles appear. Some are dispersed while others persist and diminish the overall quality of the finished product. This study consists in detecting bubbles during the resin transfer molding (RTM) process using an original method based on ultrasound. However, in practice we have to face with several problems due to the heterogeneity of the environment, such as the differentiation of a bubble from a strand or fiber. Due to the complexity of the problem, an experimental setup was built in order to detect bubbles using an ultrasonic phased array transducer in a flow of viscous fluid only, without the presence of fiber matrix. The study deals with the flow of a model fluid, which simulates the injected resin, with presence of air bubbles trapped in a channel production. Since the ultrasonic characteristics of the experimental setup are well known and characterized, the number, velocity and size of air bubbles could be evaluated.
\end{abstract}

Keywords: ultrasound, phased array, bubble, RTM process.

\section{Introduction}

The presence of bubbles in a composite material during production is the source of degradation of its expected mechanical properties. In this study, a high resolution ultrasonic phased array transducer is used for non destructive testing (NDT). More precisely, its aim is to understand the appearance and the evolution of bubbles during the manufacturing process. In recent years, phased array transducers have been increasingly used in industry for nondestructive evaluation (NDE). Indeed, high frequency electronics allows developing an excitation to generate the high resolution ultrasonic beam. This technology offers the possibility to modify parameters such as the angle of incidence, the focal distance or the size of the focus, which improves the sensitivity and effectiveness of control [1]. In addition, the beam can be shifted along the transducer, creating a sweep along the elements of the transducer. This capability opens a range of new possibilities, such as the ability to vary the angle in order to scan a sample or a weld without having to move the transducer [2]. In particular, phased array transducers are able to detect and measure velocities and size of bubbles in a viscous fluid flow, which require a very precise scan in space and time. In medicine, the technology of velocity flaw measurement already exists, but it is based on Doppler imaging [3]. Bubble detection methods are well- known in fluids and often used in water. These methods are based on bubble resonance characterization $[4,5]$. Nevertheless, the proposed method is based on a simple differentiation of ultrasound images to measure bubble sizes and velocities. In order to measure these parameters, an experimental device adapted to the real conditions of RTM process is proposed. This study is divided into four parts: the first part provides an introduction to the use of phased array; the second examines the conditions of bubble detection; the third part describes the experimental configuration; in the fourth part, the measurement results of bubble sizes and velocity are presented. 


\section{Theory}

\subsection{Phased array transducer}

To generate a beam, the phased array provides a multitude of possibilities: each element of the transducer can be excited separately, either in phase or with a phase shift. By controlling precisely the number of elements and phase shift between them, a beam can be designed with various source sizes, steering angles, and focal distances. In the case of a focus at a given point, the echo from the focal point reaches the desired elements of the transducer with a computable delay [6]. The echo signals received by each element of the transducer are then readjusted in time and then summed up. The sum for each emission/reception constitutes a time response signal, so-called an A-scan.

The used phased array transducer is made up of $N_{T}=128$ elements, with a central frequency $f_{0}=10$ MHz. The element size is $a=0.25 \mathrm{~mm}$ width by $b=7 \mathrm{~mm}$ length, and the pitch (inter-element spacing) is $d=0.5 \mathrm{~mm}$. The generated beam is a focused beam using $N=32$ elements for each focal law and with an offset of $N_{S}=1$ element between each focal law. This configuration gives a number of focal laws $N_{F L}=\left(N_{T}-N\right) / N_{S}+1=97$.

To focus the ultrasound beam at a focal depth $F$, in a bi-layer medium, here a glass plate of thickness $L_{G}$, and an oil layer of thickness $L_{O}$, it is necessary to determine the appropriate delay law to obtain the expected result [6]. Here, as illustrated by (Figure 1 (a)), we consider a linear source along the $x$ axis, focusing at the position $\left(0, L_{G}+L_{O}\right)$. More particularly, the ray source at the coordinates $\left(x_{1}, 0\right)$, (where $x_{1}$ is the central position of the farthest element from the center of the active aperture) follows Fermat's principle which consists in a minimization of the propagation time. In order to calculate the delay law of a phased array source, this principle has to be solved for each emitting element.

According to Figure 1 (a), $\cos \left(\theta_{G}\right)=L_{G} / d_{G}$ and $\cos \left(\theta_{O}\right)=L_{O} / d_{O}$, where $L_{G}$ and $d_{G}$ are the thickness and the propagation path in the glass and $L_{O}$ and $d_{O}$ are the thickness and the propagation path in the oil. The angles $\theta_{G}=\operatorname{Arctan}\left(L_{1} / L_{G}\right)$ and $\theta_{O}=\operatorname{Arctan}\left(x_{1}-L_{1} / L_{O}\right)$ are the wavefront incidence angles at position $\left(x_{1}, 0\right)$ in the glass and in the oil, respectively, and $L_{l}$ is the distance between $x_{1}$ and the incident point in the glass. The propagation times are defined as $t_{G}=d_{G} / c_{L}$,glass in the glass and $t_{O}=d_{O} / c_{\text {oil }}$ in the oil where $c_{L, \text { glass }}$ and $c_{o i l}$ are the longitudinal wave velocities in the glass and in the oil, respectively.

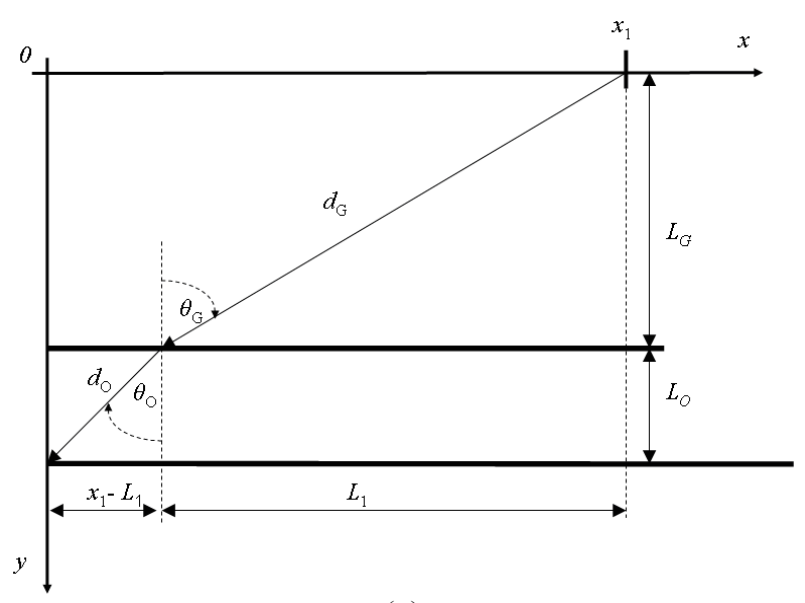

(a)

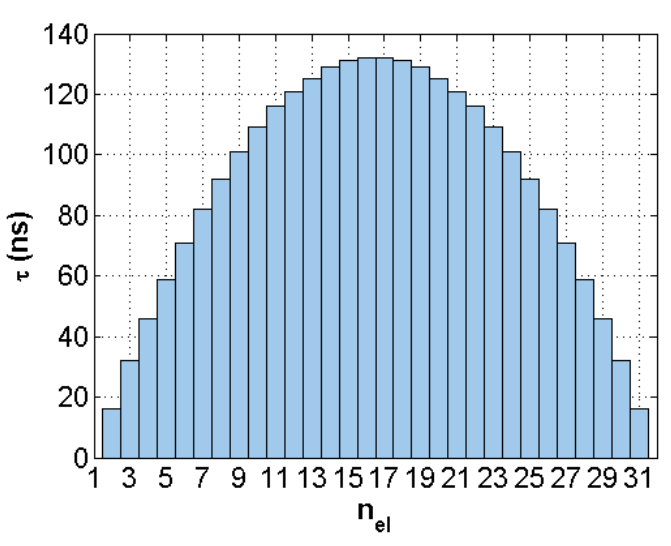

(b)

Figure 1. Delay law (a) illustration in the bi-layer structure glass-oil at normal incidence and fluid bottom focal depth and (b) calculation as a function of element number $n_{e l}$.

In order to minimize the propagation time (from emission until reaching the focal point) one should minimize the path from $\left(x_{1}, 0\right)$ to $\left(0, L_{G}+L_{O}\right)$ (Figure 1 (a)) $t\left(n_{e l}=1\right)=\left(t_{G}+t_{O}\right)$, as follows: 


$$
t\left(n_{e l}=1\right)=\frac{\sqrt{L_{G}^{2}+L_{1}^{2}}}{c_{L, g l a s s}}+\frac{\sqrt{L_{O}^{2}+\left(x_{1}-L_{1}\right)^{2}}}{c_{\text {oil }}}
$$

We want to minimize the propagation time $t\left(n_{e l}=1\right)$ relative to $L_{1}$ according to Fermat's principle $\frac{\partial t\left(n_{e l}=1\right)}{\partial L_{1}}=0$. The minimization is reduced to a $4^{\text {th }}$ order polynomial:

$L_{1}^{4}+L_{1}^{3}\left(-2 x_{1}\right)+L_{1}^{2}\left(L_{O}{ }^{2}+x_{1}{ }^{2}\right)+L_{1}\left(2 x_{1} L_{G}{ }^{2} \frac{c_{L, \text { glass }}}{c_{\text {oil }}-c_{L, \text { glass }}}\right)-\frac{x_{1}{ }^{2} L_{G}{ }^{2} c_{L, \text { glass }}}{c_{\text {oil }}-c_{L, \text { glass }}}=0$

Once $L_{1}$ is determined, the propagation time $t\left(n_{e l}\right)$ relative to the $n^{\text {th }}$ element $n_{e l}$, with $1 \leq n_{e l} \leq N$, can also be determined. In this aim, the position $x_{1}$ relative to the emitter 1 is generalized to other positions of emitters $n_{e l}$ by $\left(x_{1}-\left(n_{e l}-1\right) d\right)$, where $d$ is the pitch.

We obtain the following equation:

$$
t\left(n_{e l}\right)=\frac{\sqrt{L_{G}^{2}+L_{1}^{2}}}{c_{L, \text { glass }}}+\frac{\sqrt{L_{O}^{2}+\left(x_{1}-\left(n_{e l}-1\right) d-L_{1}\right)^{2}}}{c_{\text {oil }}}
$$

The solutions of Eq. (3) are solved analytically using Matlab code. The physical solution $L_{1}$ is the one which provides the optimal path delay. Eventually, the electronic excitation delay $\tau\left(n_{e l}\right)$ applied to the element $n_{e l}$ is:

$$
\tau\left(n_{e l}\right)=t\left(n_{e l}=1\right)-t\left(n_{e l}\right)
$$

The representation of $\tau\left(n_{e l}\right)$ for $L_{G}=40 \mathrm{~mm}$ and $L_{O}=3 \mathrm{~mm}$ is given by (Figure 1 (b)).

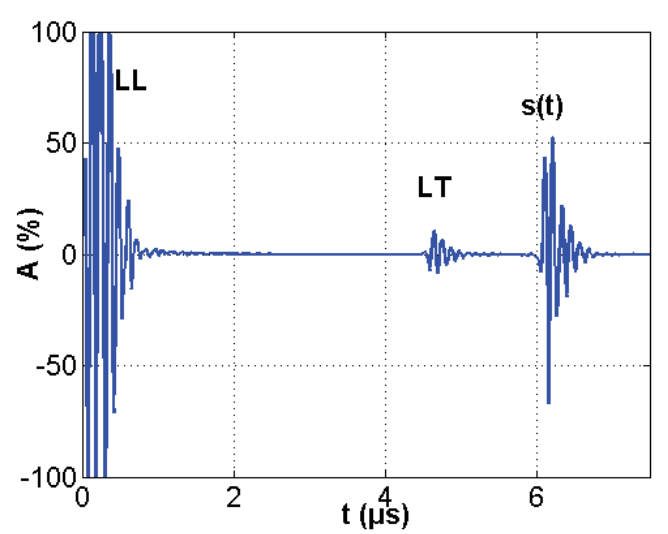

Figure 2. Time response (A-scan) of the focused beam emitted into the fluid without macro-bubbles with (LL) longitudinal wave in the glass used as time reference, (LT) longitudinal to transversal mode conversion in the glass and $s(t)$ back echo from the fluid.

This delay law corresponds to a focal law and every focal law provides an A-scan. The A-scan is a time response signal (Figure 2) resulting from a focus on the bottom of the fluid layer using $N=32$ elements. Consequently, mode conversions on the back face of the glass appear in two forms: a conversion from a longitudinal wave to a transversal wave denoted (LT) or from a transversal to a longitudinal one denoted (TL). In the two cases, the signals from these conversion modes arrive at the same date, so we limit the notation to (LT). Also, the longitudinal wave travelling in the glass without conversion modes is denoted (LL) and used as the reference time. The useful signal reflected on the bottom of the fluid layer is denoted $s(t)$ (Figure 2). The time of flight after a roundtrip in the fluid is $t=6.25 \mu \mathrm{s}$. Knowing the thickness of the fluid layer $L_{O}=3 \mathrm{~mm}$, we obtain a sound velocity $c_{\text {oil }}=960 \mathrm{~m} / \mathrm{s}$ in agreement with preliminary studies [7]. This helps to assess the longitudinal $c_{L, \text { glass }}=5550 \mathrm{~m} / \mathrm{s}$ and transverse $c_{T, \text { glass }}=3350 \mathrm{~m} / \mathrm{s}$ wave velocity values in the glass. The thickness of the glass plate was chosen so that the echoes from waves having made roundtrips in the glass and round trips in the fluid are clearly separated as well as $t_{S(t)}>t_{L T}$, where $t_{s(t)}$ is the date of back echo $\left(t_{L L}+2 L_{O} / c_{o i l}\right)$ and $t_{L T}$ the date of the longitudinal-transversal conversion mode in 
the glass plate. Also, the thickness of the aluminum plate was chosen so that it would be thick enough to delay the back echoes and to avoid interferences.

\subsection{Resolution of a focused phased array transducer}

The detection condition of an air bubble in a viscous fluid by an ultrasonic wave is the occurrence of a disturbance of the wavefront caused by the impedance ratio between oil and air. The amplitude of the back face echo of oil is affected by the wave diffraction on the bubble; thus, the bubble becomes visible by a decrease of the $s(t)$ echo amplitude. However, this condition is satisfied only when the bubble radius $r_{\text {bubble }}$ is significant in comparison to the wavelength in the fluid $\lambda_{\text {fluid }}$ and should fulfill the condition $r_{b u b b l e} / \lambda_{\text {fluid }} \geq 1$. Also the reflection coefficient $|R|$ (Eq. (6)) between the fluid and the air bubble has to be nearly equal to 1 if we neglect the air acoustic impedance $Z_{\text {air }}$ compared to that of the fluid $Z_{\text {fluid }}$ :

$$
|R|=\left|\frac{Z_{\text {air }}-Z_{\text {fluid }}}{Z_{\text {air }}+Z_{\text {fluid }}}\right| \approx 1
$$

where $Z_{\text {air }}=\rho_{\text {air }} c_{\text {air }}=340 \mathrm{Ra}$ and $Z_{\text {fluid }}=\rho_{\text {fluid }} \mathcal{c}_{\text {fluid }}=1 \mathrm{MRa}$ with $\rho_{\text {air }}$ and $c_{\text {air }}$ are the density and the wave velocity in the air, and $\rho_{\text {fluid }}$ and $c_{\text {fluid }}$ the density and wave velocity in the fluid, respectively. In this study, the transducer was chosen for high resolution purpose at $f_{0}=10 \mathrm{MHz}$. It emits in a silicone oil fluid having a wave velocity of around $c_{\text {fluid }} \approx 1000 \mathrm{~m} / \mathrm{s}$, resulting in $\lambda_{\text {fluid }}=c_{\text {fluid }} / f_{0} \approx 100$ $\mu \mathrm{m}$. The inequality $r_{\text {bubble }} / \lambda_{\text {fluid }} \geq 1$ sets the lower limit of detection around $r_{\text {bubble }} \approx 100 \mu \mathrm{m}$. Below this value, it is called micro-bubble, and beyond, we talk about macro-bubble. Now, we will look at the elementary volume information produced by a focal law in order to estimate the size of bubbles that can be observed on the $\mathrm{C}$-scan image. The volume resolution is a voxel of dimensions ( $\Delta x, \Delta y$, $\Delta z$ ). The echo duration of $s(t)$ (Figure 2) along the $z$ axis (wave propagation axis) is calculated on the pulse-echo response at $-6 \mathrm{~dB}$, denoted $t_{-6} \mathrm{~dB}$ as follows:

$$
\Delta z=\frac{c_{o i l} \cdot t_{-6 \mathrm{~dB}}}{2}=96 \mu \mathrm{m} \approx 100 \mu \mathrm{m}
$$

These dimensions are estimated by the directivities $D_{x}\left(\theta_{x}\right)$ and $D_{y}\left(\theta_{y}\right)$ as a function of the angular resolution $\theta_{x, y}$ along the $x$ axis (probe length axis) and $y$ axis (probe width axis), respectively. According to the Huygens principle, the directivity results from the spherical superposition of elementary contributions. In emission-reception, the pressure at the receiving point is the product of two contributions (emission and reception). This phased array is considered as a linear array consisting of single element sources, and each element source is approximated as an assembly of infinitely large number of simple line sources arranged in the length probe direction [8]. Azar [6] compared this configuration in agreement with a transducer of equally spaced array of simple sources since the element width $a$ is small compared to the wavelength in the fluid $\lambda_{\text {fluid }}$ [9].

The directivity along $x$ axis $D_{x}\left(\theta_{x}\right)$ is given by Azar [6] as follows:

$$
D_{x}\left(\theta_{x}\right)=\left|\frac{\sin \left(\frac{k a \sin \theta_{x}}{2}\right)}{\frac{k a \sin \theta_{x}}{2}} e^{j \frac{k a \sin \theta_{x}}{2}} \cdot\left(\sum_{n=0}^{N-1} e^{j\left(n_{e l} k a \sin \theta_{x}-\omega \tau\left(n_{e l}\right)\right.}\right)\right|
$$

where $a$ is the element width, $k=\omega / c_{\text {fluid }}$ is the wave number, $\omega$ is the angular frequency, $c_{f l u i d}$ is the wave velocity in the fluid and $\tau\left(n_{e l}\right)$ is the delay law given by Eq. (4).

Similarly, the directivity $D_{y}\left(\theta_{y}\right)$ along the $y$ axis is:

$$
D_{y}\left(\theta_{y}\right)=\left|\frac{\sin \left(\frac{k b \sin \theta_{y}}{2}\right)}{\frac{k b \sin \theta_{y}}{2}}\right|
$$

where $b$ is the length of an element. 


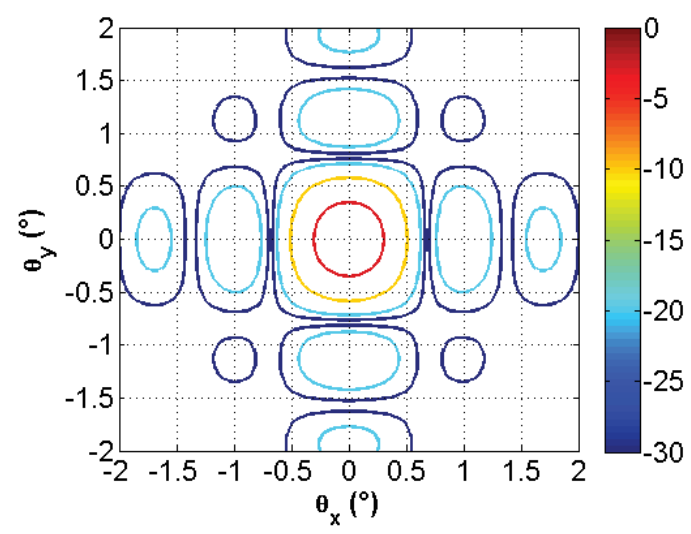

Figure 3. Angular directivities along $x$ and $y$ axis in the bi-layer structure for a focused phased array transducer using 32 elements at normal incidence: isovalues are given in red, yellow, cyan and blue at $-3,-10,-20$ and $-30 \mathrm{~dB}$, respectively.

The angular resolutions at $-3 \mathrm{~dB}$ computed from $\mathrm{Eq}(8,9)$ along the $x$ and $y$ axes are nearly the same and evaluated at $\theta_{x,-3 \mathrm{~dB}}=0.27^{\circ} \approx 4.71 \mathrm{mrad}$ and $\theta_{y,-3 \mathrm{~dB}}=0.31^{\circ} \approx 5.50 \mathrm{mrad}$ respectively. This result (Figure 3) is obtained for a focused phased array transducer using $N=32$ elements at normal incidence. The $\mathrm{dB}$ level gives isovalues at different $-n \mathrm{~dB}$ level, where $n$ is a real number $0 \leq n \leq 30$ $\mathrm{dB}$. This figure highlights secondary and tertiary lobes around $-12 \mathrm{~dB}$ and $-18 \mathrm{~dB}$, respectively. The angular resolution is used to calculate lateral resolutions $\Delta x$ and $\Delta y$ expressed as follows:

$$
\begin{aligned}
& \Delta x=2 F \tan \left(\theta_{x,-3 \mathrm{~dB}}\right)=93 \mu \mathrm{m} \\
& \Delta y=2 F \tan \left(\theta_{y,-3 \mathrm{~dB}}\right)=109 \mu \mathrm{m}
\end{aligned}
$$

where $F=L_{O}+L_{G} c_{\text {oil }} / c_{\text {glass }}$ is the equivalent focusing depth in the oil corresponding to the two propagation mediums [10].

As a result, the resolution along the three dimensions is about $100 \mu \mathrm{m} \pm 10 \%$ and the volume resolution can be considered as a cube of $100 \mu \mathrm{m}$ wide. This result allows us to estimate the smallest detectable bubble size.

\section{Experiment}

\subsection{Set-up}

The objective of this study is to detect macro-bubbles in a viscous fluid flow. The chosen fluid is a silicone oil with a dynamic viscosity $\eta=50 \mathrm{mPa}$.s. Using a pump it is injected into a channel between two plates, one of glass and one of aluminum to simulate the RTM mold. Air bubbles are injected into the flow through a valve that allows the aspiration of air at the entry of the fluid (Figure 4 (a)). The phased array transducer is in contact with the glass plate, here considered as a wedge, so the (LL) echo provides the time reference. The wave propagates in the glass and then focuses in the fluid at the fluid-aluminum interface. The back echoes from the aluminum plate are sufficiently delayed relative to those reflected at the oil-aluminum interface and do not interfere with the observed response $s(t)$ (Figure 2). 


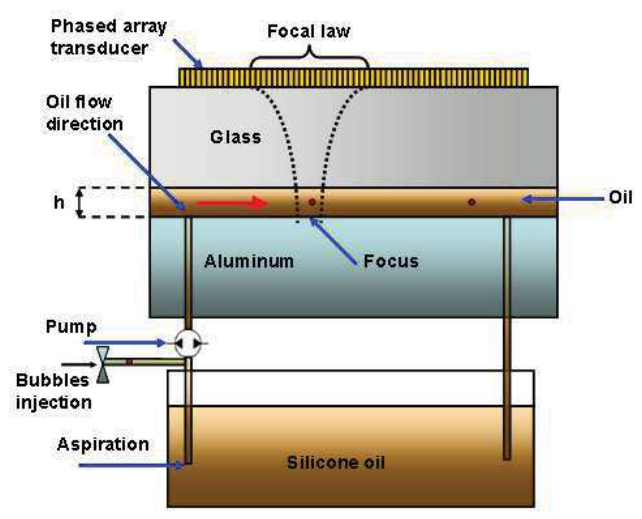

(a)

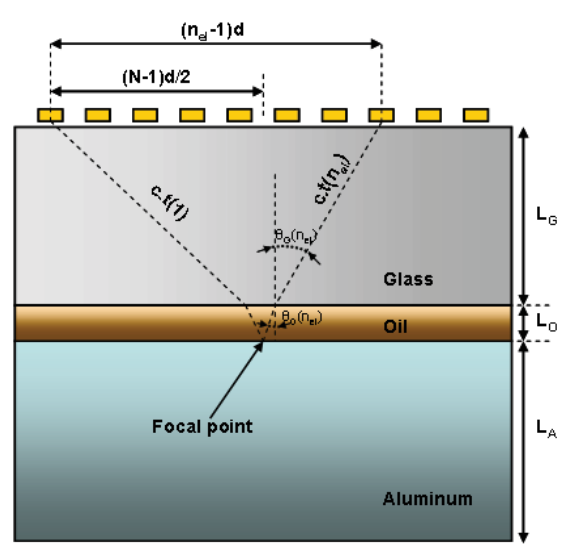

(b)

Figure 4. (a) Set-up for bubble monitoring (b) Geometry and parameters involved in the focus of the phased array: $N=$ $32, d=0.5 \mathrm{~mm}, L_{G}=40 \mathrm{~mm}, c_{L, \text { glass }}=5550 \mathrm{~mm} / \mathrm{s}, L_{O}=3 \mathrm{~mm}$ and $c_{\text {oil }}=960 \mathrm{~mm} / \mathrm{s}$.

\subsection{Data acquisition}

The active aperture $X=63.75 \mathrm{~mm}$ is the total active probe length:

$$
X=\left(N_{T}-1\right) d+a
$$

where $N_{T}$ is the total number of elements constituting the transducer, $d$ is the pitch and $a$ is the element width. Since each focal law provides an A-scan, the position of the focus relative to each focal law compared to the active probe length is determined as follows:

$$
x_{F L}\left(n_{F L}\right)=\left[\left(\frac{N-1}{2}\right)+\left(n_{F L}-1\right) N_{s}\right] d
$$

where $1 \leq n_{F L} \leq N_{F L}$ is the focal law number, $N_{F L}$ is the total number of focal laws, $N$ is the number of elements per focal law (Figure 4(b)) and $N_{s}=1$ element is the offset between two focal laws.

According to Eq. (12), we deduce the coordinate range of focal laws $x_{F L}(1)=7.75 \mathrm{~mm}$ up to $x_{F L}\left(N_{F L}\right)=55.75 \mathrm{~mm}$ and the total width of the beam is $X_{F L}=\left(N_{T}-N\right) . d=48 \mathrm{~mm}$.

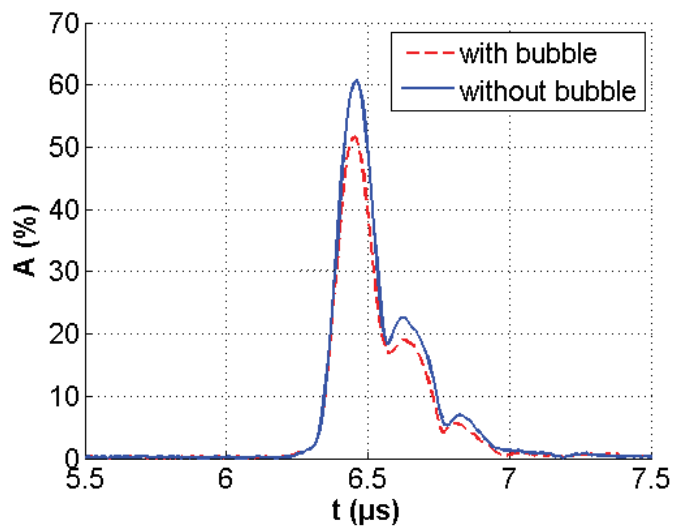

Figure 5. Comparison of the envelope of the echo amplitude $s(t)$ without (blue solid) and with macro-bubbles (red dashed) in the fluid.

Air bubbles generated by aspiration cross the beam along its width with different velocities. Bubbles are visible on the envelope of the back echo $s(t)$ by a small decrease in the amplitude (Figure 5) due to diffraction by the bubble.

The C-scan image $s\left(x_{\mathrm{FL}}, t_{a c q}\right)$ is shaped by concatenating the maximum of the envelope of the Ascan $e(t)$ calculated using the Hilbert transform in the observation window from 5.5 to $7.5 \mu \mathrm{s}$ (Figure 5). This operation is done for each focal law (position) and for each acquisition date (time). One acquisition is a sweep of all focal laws, and each acquisition is refreshed at a sampling frequency $f_{a c q}=20 \mathrm{~Hz}$. A date $t_{a c q}=\left(n_{a c q}-1\right) / f_{a c q}$ is associated to each acquisition $n_{a c q}$ where $1 \leq n_{a c q}$ 
$\leq N_{a c q}$. The total number of acquisitions is chosen $N_{a c q}=201$. Thus, the necessary time for a C-scan is $T_{a c q}=\left(N_{a c q}-1\right) / f_{a c q}=10 \mathrm{~s}$.

Finally, the obtained C-scan image is composed of $N_{F L} \times N_{a c q}=97 \times 201$ points with coordinates $\left(x_{F L}\left(n_{F L}\right), t_{a c q}\left(n_{a c q}\right)\right), x_{F L}=0$ to $X_{F L}=48 \mathrm{~mm}$, and $t_{a c q}=0$ to $T_{a c q}=10 \mathrm{~s}$.

\section{Results}

\subsection{Estimation of bubbles quantity and velocities}

Now, to make the bubbles appear more clearly (Figure 6 (a)), the C-scan image is processed. Image processing consists in subtracting the average of the C-scan image. $s_{\text {mean }}$ is the mean of $s\left(x_{F L}, t_{a c q}\right)$ for all the acquisition positions $x_{F L}$ and dates $t_{a c q}$. Thus, the processing results in: $s_{2}\left(x_{F L}, t_{a c q}\right)=s_{\text {mean }}$ $-s\left(x_{F L}, t_{a c q}\right)$.

Considering the position $x_{F L}=24.5 \mathrm{~mm}$ during the acquisition time $T_{a c q}=10 \mathrm{~s}$ (Figure 6(a)) and comparing with the $\mathrm{C}$-scan image, we can deduce that the lines with greater contrasts correspond to higher amplitudes, i.e. a high volume fraction of bubbles. In this case, the maximum amplitudes are given at $t_{a c q}=0.15$ and $9.6 \mathrm{~s}$.

We can also determine the number of bubbles crossing the ultrasound beam at the position $x_{F L}=$ $24.5 \mathrm{~mm}$ by counting the summits exceeding the threshold fixed at twice the average noise level.

The bubbles are visible on the processed C-scan image $s_{2}\left(x_{\mathrm{FL}}, t_{a c q}\right)$ by lines of different slopes. As shown in Figure 6 (a), the bubbles crossing the phased array focal law position $x_{F L}$, for a given acquisition time $t_{a c q}$, are detected at the associated coordinates $\left(x_{F L}\left(n_{F L}\right), t_{a c q}\left(n_{a c q}\right)\right)$. The bubble velocity $v_{b}=\Delta x_{F L} / \Delta t_{a c q}$ is then determined from the slopes (Figure 6 (a)).

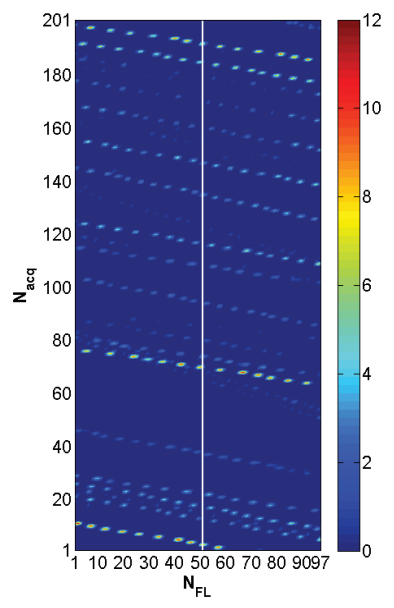

(a) $24.5 \mathrm{~mm}$ (white solid line) and maximum of amplitudes (red circles) with a total number of bubbles $N_{b}=20$ and (b) bubble velocity distribution $\left(n_{b}, v_{b}\right)$ with a mean velocity $v_{b, \text { mean }}=54 \mathrm{~mm} / \mathrm{s}$.

The velocity of each bubble $v_{b}$ is thus computed using image processing. As a result, the velocity distribution of the bubbles (number of bubbles $N_{b}$ as function of bubble velocity $v_{b}$ ) is presented in (Figure 6 (b)) with its average value $v_{b, \text { mean }}=54 \mathrm{~mm} / \mathrm{s}$. The bubble velocities are measurable in a range of values fixed by the global processing unit. The lower velocity boundary that can be detected is equal to:

$$
v_{\min }=\frac{d . f_{a c q}}{N_{a c q}-1}=\frac{0.5 \times 20}{200}=0.05 \mathrm{~mm} / \mathrm{s}
$$

and the upper velocity boundary is equal to:

$$
v_{\max }=X_{F L} \cdot f_{a c q}=48 \times 20=960 \mathrm{~mm} / \mathrm{s}
$$




\subsection{Validation of bubble velocities and sizes estimations}

In this part, in order to validate ultrasonic phased array measurements, we compared the size and velocity of phased array measurements to video captures and to the theoretical model. In another configuration the glass plate and the aluminum plate are replaced by two plates of PMMA to ensure the transparency for video capture on both sides. Calibrated bubbles are injected into the transparent mold and their evolution is monitored by a camera on one side and by the phased array transducer on the other side. The mold is positioned vertically and calibrated bubbles evolve from the bottom to the top of the mold according to Archimedes' principle and viscous forces. As a solution of this differential equation, the limit ascent velocity of bubble $v$ and time constant $\tau$ are depending on the drag coefficient $C_{g}$. This drag coefficient depends on the Reynolds number $R_{e}=v D \rho_{\text {fluid }} / \eta_{\text {fluid }}$, a weighting factor $A=(1+2 \alpha / 3) /(1+\alpha)$ depending itself on $\alpha=\eta_{\text {fluid }} / \eta_{\text {air }}$ [11] (Table 1). The Stokes model [12] is given for a rigid interface (solid sphere in a fluid) i.e. $\alpha \rightarrow 0$; however Hadamard (fluid sphere in a fluid) [13] and Keh \& Shiau (fluid sphere in a fluid) [14] models are used whatever the $\alpha$ parameter. In our case $\alpha$ can be considered as $\alpha \rightarrow \infty$.

Table 1: Time constant $\tau$ and drag coefficient $C_{g}$ : Stokes [12], Hadamard [13] and Keh and Shiau [14] models.

\begin{tabular}{|c|c|c|c|}
\hline Reference & Stokes [12] & Hadamard [13] & Keh and Shiau [14] \\
\hline$\tau(\mathrm{s})$ & $D^{2} \rho_{\text {air }} /\left(18 \eta_{\text {fluid }}\right)$ & $D^{2} \rho_{\text {air }} /\left(18 A \eta_{\text {fluid }}\right)$ & - \\
\hline$C_{g}$ & $\frac{24}{R_{e}}$ & $\frac{24}{R_{e}} A$ & $\frac{24}{R_{e}} A .\left(1+\frac{3}{8} A^{2} R_{e}+\frac{9}{40} R_{e}^{2} \ln \left(R_{e}\right)\right)$ \\
\hline $\begin{array}{l}D \text { : bubble diameter; } \\
\text { gravity constant. }\end{array}$ & dynamic viscosity of the fluid; $\rho_{\text {fluid }}:$ density of the fluid; $\rho_{\text {air }}:$ density of the air; $g$ : \\
\hline
\end{tabular}

The velocity is derived from the Stokes's and Hadamard's equations as follows:

$$
v=\tau \frac{\rho_{\text {fluid }}-\rho_{\text {air }}}{\rho_{\text {air }}} g
$$

The Keh and Shiau equation needs to solve numerically a differential equation of the form: $d v / d t=K_{0}+K_{1} v+K_{2} v^{2}+K_{3} v^{3} \cdot \ln (N v)$, where $K_{0}, K_{1}, K_{2}, K_{3}$ and $N$ are constants.

The results are summarized as curves $(D, v)$ in (Figure 7 (a)). One can observe that the three methods (Keh \& Shiau, Video, Ultrasound) are in agreement for a flow with low Reynolds numbers: $0.3<R_{e}<2$. Stokes [12] and Hadamard [13] models are only valid for small Reynolds number: $R_{e}<<1$. However, Keh \& Shiau [14] take into account the higher order terms $\left(\sim R_{e}^{2}\right)$ (Table 1). Moreover, the wall effect is taken into account for these different models, with the wall correction factor extrapolated from Haberman model [15]. 

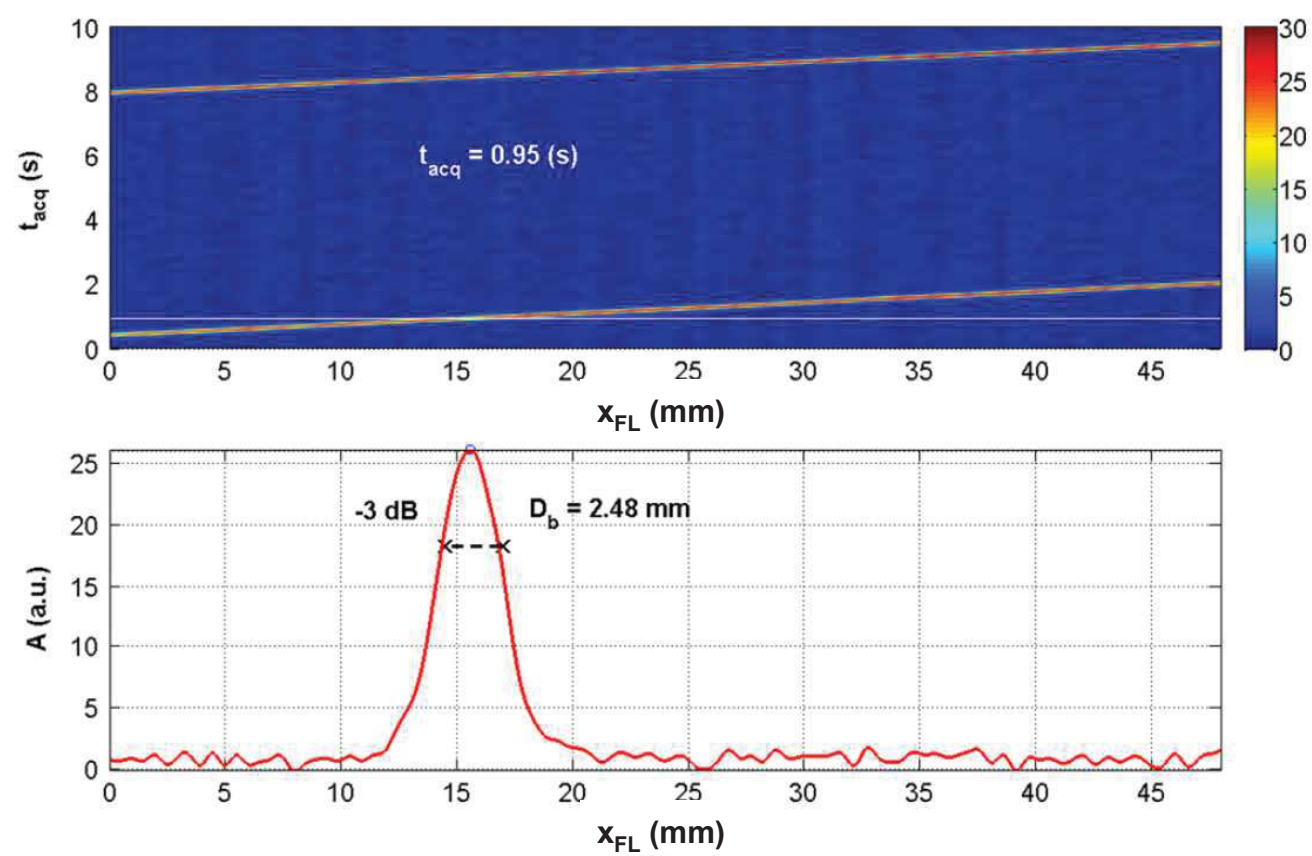

(b)

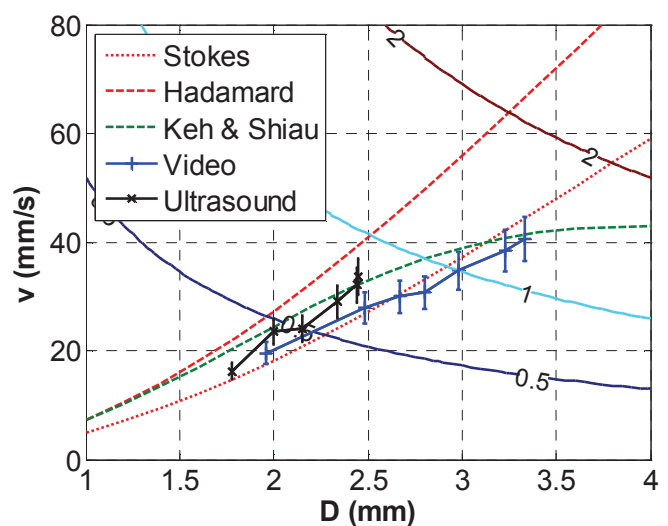

(a)

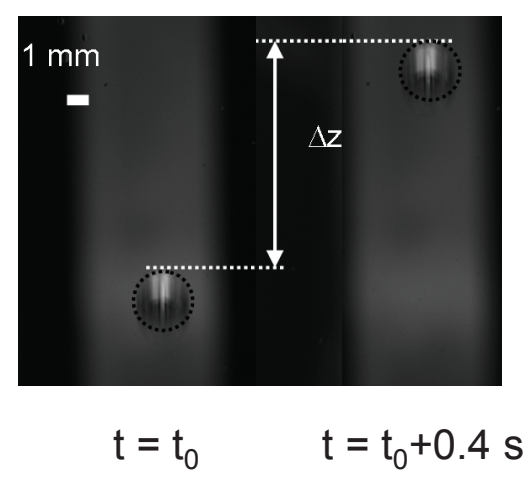

(c)

Figure 7. Validation of phased array size and velocity measurements (a) $(D, v)$ using theoretical Stokes, Hadamard and Keh and Shiau models, and ultrasound and video measurements for $0.2 \leq R_{e} \leq 2$, using calibrated bubbles and (b) processed ultrasound C-scan giving $\left(D_{u s}, v_{u s}\right)$ and (c) processed video giving $\left(D_{\text {video }}, v_{\text {video }}\right)$.

The measurements are given for seven bubbles of different diameters; the velocity presented here at $\pm 10 \%$ is increasing to the squared diameter of the bubble. The ultrasound measurement of bubble diameter is fixed after calibration at $-3 \mathrm{~dB}$ of the maximum of the reflected signal on the bubble (Figure 7 (b)). This measurement is made at fixed $t_{a c q}$ and for all the positions $x_{F L}$ (Figure 7 (b)). As illustrated by (Figure 7 (c)) the diameter given by the camera is evaluated on a frame and the velocity is determined between two different time delays $t_{0}$ and $t_{0}+\Delta t$ for which the bubble traveled a distance $\Delta z$.

\section{Conclusion}

This study consisted of modeling the RTM process and monitoring bubbles in the resin flow through an ultrasonic phased array transducer. An experimental device was proposed to monitor the evolution of bubbles in a viscous fluid flow. First, the velocity of macro-bubbles was measured over a given period of acquisition set by the global processing unit, i.e. hardware and software limitations. Then, bubble quantity, and differentiation of their sizes was observed. Finally, the reliability of ultrasound diameter and velocity measurement was validated by correlation with video measurements and theoretical results. 


\section{Acknowledgements}

This work is supported by the CNRS and the Région Haute Normandie.

\section{References}

[1] Wooh S, Shi Y. Influence of phased array element size on beam steering behavior. Ultrasonics 1998; 36:737-749.

[2] Huang R, Schmerr L. Characterization of the system functions of ultrasonic linear phased array inspection systems. Ultrasonics 2009; 49:219-225.

[3] Jensen J. Algorithms for estimating blood velocities using ultrasound. Ultrasonics 2000; 38 : $358-362$.

[4] Leighton T. Comparison of the abilities of eight acoustic techniques to detect and size a single bubble. Ultrasonics 1996; 34:661-667.

[5] Leighton TG. The effect of reverberation on the damping of bubbles. J Acoust Soc Am. 2002; 112 (4):1366-1376.

[6] Azar L, Shi Y, Wooh S. Beam focusing behavior of linear phased arrays, NDT E Int 2000; 33:189-198.

[7] Samet N, Marechal P, Duflo H. Détermination de l'atténuation ultrasonore d'une huile silicone et estimation de la viscosité in Proceeding of 10ème Congrès Français d'Acoustique conference, Lyon, 2010.

[8] Wooh S, Shi Y. Optimum beam steering of linear phased arrays. Wave Motion 1999; 29: 245-265.

[9] Buchana M, Hynynen K. Design and experimental evaluation of an intracavity ultrasound phased array system for hyperthermia. IEEE Trans Biomed Engng Design 1983; 76 (3): $275-283$.

[10] Jeong H, Hsu D. Experimental analysis of porosity-induced ultrasonic attenuation and velocity change in carbon composites. Ultrasonics 1995; 3: 195-203.

[11] Samet N, Marechal P, Duflo H. Détection de bulles en mouvement dans une préforme fibreuse par méthode ultrasonore in Proceeding of Congrès Français de Mécanique, Besançon 2011.

[12] Lamb H. Hydrodynamics (6th edition ed.), 1994, Cambridge University Press.

[13] Hadamard J. Mouvement permanent lent d'une sphère liquide et visqueuse dans un liquide visqueux, C. R. Acad. Sci. 1911, 152, 1735.

[14] Keh HJ, Shiau SC. Effects of inertia on the slow motion of aerosol particles, Chemical Engineering Science 2000, 55, 4415-4421.

[15] Haberman WL, Sayre RM. Motion of rigid and fluid spheres in stationary and moving liquids inside cylindrical tubes, October 1958. 